University of Wollongong

Research Online

Faculty of Social Sciences - Papers (Archive) Faculty of Arts, Social Sciences \& Humanities

2019

Interventions in outside-school hours childcare settings for promoting physical activity amongst schoolchildren aged 4 to 12 years

Rosa Virgara

University of South Australia

Carol Maher

University of South Australia

Lucy Lewis

Flinders University

Anna Phillips

University of South Australia

Luke Wolfenden

University of Newcastle

See next page for additional authors

Follow this and additional works at: https://ro.uow.edu.au/sspapers

Part of the Education Commons, and the Social and Behavioral Sciences Commons

Research Online is the open access institutional repository for the University of Wollongong. For further information contact the UOW Library: research-pubs@uow.edu.au 


\title{
Interventions in outside-school hours childcare settings for promoting physical activity amongst schoolchildren aged 4 to 12 years
}

\author{
Abstract \\ This is a protocol for a Cochrane Review (Intervention). The objectives are as follows: The primary \\ objective is to assess the effects of physical activity interventions for increasing physical activity in \\ children aged 4 to 12 years in outside-school hours childcare settings (i.e. the hours of the day when \\ formal school lessons have either not begun or have finished and/or during school holiday periods). Our \\ secondary objectives are to assess the effects of interventions on markers of children's cardiovascular \\ health, quality of life, process evaluation and to identify any unintended adverse outcomes (e.g. injuries, \\ distress).

\section{Disciplines} \\ Education | Social and Behavioral Sciences

\section{Publication Details} \\ Virgara, R., Maher, C., Lewis, L. K., Phillips, A., Wolfenden, L., Okely, A., Beets, M. W., Richardson, M. \& \\ Baldock, K. (2019). Interventions in outside-school hours childcare settings for promoting physical activity \\ amongst schoolchildren aged 4 to 12 years. Cochrane Database of Systematic Reviews, 2019 (7), 1-17.

\section{Authors} \\ Rosa Virgara, Carol Maher, Lucy Lewis, Anna Phillips, Luke Wolfenden, Anthony D. Okely, Michael W. Beets, \\ Mandy Richardson, and Katherine Baldock
}




\section{(E) Cochrane Library}

Cochrane Database of Systematic Reviews

\section{Interventions in outside-school hours childcare settings for promoting physical activity amongst schoolchildren aged 4 to 12 years (Protocol)}

Virgara R, Maher C, Lewis LK, Phillips A, Wolfenden L, Okely A, Beets MW, Richardson M, Baldock K

Virgara R, Maher C, Lewis LK, Phillips A, Wolfenden L, Okely A, Beets MW, Richardson M, Baldock K.

Interventions in outside-school hours childcare settings for promoting physical activity amongst schoolchildren aged 4 to 12 years.

Cochrane Database of Systematic Reviews 2019, Issue 7. Art. No.: CD013380.

DOI: 10.1002/14651858.CD013380.

www.cochranelibrary.com 


\section{TABLE OF CONTENTS}

HEADER . . . . . . . . . . . . . . . . . . . . . . . . . . . . . . . . . . . . . . . . 1

ABSTRACT . . . . . . . . . . . . . . . . . . . . . . . . . . . . . . . . . . . . . . . 1

BACKGROUND . . . . . . . . . . . . . . . . . . . . . . . . . . . . . . . . . . . . . . . 1

OBJECTIVES . . . . . . . . . . . . . . . . . . . . . . . . . . . . . . . . . . . . . . 3

METHODS . . . . . . . . . . . . . . . . . . . . . . . . . . . . . . . . . . . . . . .

ACKNOWLEDGEMENTS . . . . . . . . . . . . . . . . . . . . . . . . . . . . . . . . . . . . . . . . . . .

REFERENCES . . . . . . . . . . . . . . . . . . . . . . . . . . . . . . . . . . . . . . . . . 8

APPENDICES . . . . . . . . . . . . . . . . . . . . . . . . . . . . . . . . . . . . . . . . . . . . . . . . .

CONTRIBUTIONS OF AUTHORS . . . . . . . . . . . . . . . . . . . . . . . . . . . . . . . . . . . . . . . . . . . . 16

DECLARATIONS OF INTEREST . . . . . . . . . . . . . . . . . . . . . . . . . . . . . . . . . . . 16

SOURCES OF SUPPORT . . . . . . . . . . . . . . . . . . . . . . . . . . . . . . . . . . . . . . . 16

Interventions in outside-school hours childcare settings for promoting physical activity amongst schoolchildren aged 4 to 12 years

(Protocol)

Copyright $\odot 2019$ The Cochrane Collaboration. Published by John Wiley \& Sons, Ltd. 


\section{Interventions in outside-school hours childcare settings for promoting physical activity amongst schoolchildren aged 4 to 12 years}

Rosa Virgara ${ }^{1}$, Carol Maher ${ }^{2}$, Lucy K Lewis ${ }^{3}$, Anna Phillips ${ }^{4}$, Luke Wolfenden ${ }^{5}$, Anthony Okely ${ }^{6}$, Michael W Beets ${ }^{7}$, Mandy Richard$\operatorname{son}^{8}$, Katherine Baldock ${ }^{4}$

${ }^{1}$ Alliance for Research in Exercise, Nutrition and Activity (ARENA), School of Health Sciences, University of South Australia, Adelaide, Australia. ${ }^{2}$ School of Health Sciences, Sansom Institute, University of South Australia, Adelaide, Australia. ${ }^{3}$ College of Nursing and Health Sciences, Flinders University, Bedford Park, Australia. ${ }^{4}$ School of Health Sciences, University of South Australia, Adelaide, Australia. ${ }^{5}$ School of Medicine and Public Health, University of Newcastle, Callaghan, Australia. ${ }^{6}$ Early Start Research Institute, University of Wollongong, Wollongong, Australia. ${ }^{7}$ Arnold School of Public Health, University of South Carolina, Columbia, South Carolina, USA. ${ }^{8}$ Outside School Hours Care (OSHC) Service, St Pius X School, Windsor Gardens, Australia

Contact address: Carol Maher, School of Health Sciences, Sansom Institute, University of South Australia, City East Campus, North Terrace, Adelaide, South Australia, 5000, Australia. carol.maher@unisa.edu.au.

Editorial group: Cochrane Public Health Group.

Publication status and date: New, published in Issue 7, 2019.

Citation: Virgara R, Maher C, Lewis LK, Phillips A, Wolfenden L, Okely A, Beets MW, Richardson M, Baldock K. Interventions in outside-school hours childcare settings for promoting physical activity amongst schoolchildren aged 4 to 12 years. Cochrane Database of Systematic Reviews 2019, Issue 7. Art. No.: CD013380. DOI: 10.1002/14651858.CD013380.

Copyright (C) 2019 The Cochrane Collaboration. Published by John Wiley \& Sons, Ltd.

\section{A B S T R A C T}

This is a protocol for a Cochrane Review (Intervention). The objectives are as follows:

The primary objective is to assess the effects of physical activity interventions for increasing physical activity in children aged 4 to 12 years in outside-school hours childcare settings (i.e. the hours of the day when formal school lessons have either not begun or have finished and/or during school holiday periods). Our secondary objectives are to assess the effects of interventions on markers of children's cardiovascular health, quality of life, process evaluation and to identify any unintended adverse outcomes (e.g. injuries, distress).

\section{B A C K G R O U N D}

\section{Description of the condition}

Non-communicable diseases (NCDs), such as stroke, heart disease, type 2 diabetes, cancer, and chronic lung disease are responsible for approximately $70 \%$ of deaths worldwide (Ding 2016). The rise in the prevalence of NCDs is primarily due to four major risk factors: tobacco use, harmful use of alcohol, poor diet, and physical inactivity WHO 2018a. Lack of physical activity (PA) has been identified as a universal issue that requires global intervention (Ding 2016). Literature has demonstrated the effect of physical inactivity on morbidity and premature mortality (Ding 2016). The resultant economic burden on healthcare systems has been estimated internationally at approximately USD 53800 million, with countries with higher human development index impacted more with economic costs, whilst countries of middle to

Interventions in outside-school hours childcare settings for promoting physical activity amongst schoolchildren aged 4 to 12 years 
low human development suffer with greater disease burden (Ding 2016).

Physical activity provides an array of health benefits across the lifespan. In adulthood, PA has been linked to improved cardiovascular health and reduced rates of overweight and obesity, cancer, and other NCDs (Reiner 2013). Moderate-to-vigorous physical activity (MVPA) is activity that induces energy expenditure greater than 3 METs (metabolic equivalents), such as brisk walking, running, dancing, competitive sports, etc. (WHO 2018b). In children, MVPA is associated with many health-related benefits, including improved aerobic fitness, cognitive abilities, and self-confidence, together with reduced cardiovascular risk and depression rates (Sterdt 2014). Evidence suggests that those who participate in regular physical activity in childhood are more likely to be physically active as adults (Jones 2013; Tammelin 2014). Despite these benefits, numerous studies have found that children's physical activity levels have been declining in recent decades (Bassett 2015; Dalene 2018; Dollman 2005), with outdoor play (Bassett 2015), active transport (Bassett 2015; Booth 2015; Dollman 2005), and physical education implicated in particular (Bassett 2015; Dollman 2005). Given the wide-ranging impacts of physical activity for children's health and well-being, international guidelines have been developed.

The World Health Organization's (WHO) physical activity and sedentary behaviour guidelines for children aged 5 to 18 years recommend that children attain at least 60 minutes of MVPA and no more than two hours of recreational screen time daily (e.g. activities for recreation/enjoyment such as computer or tablet use, television viewing, and inactive video gaming, not screen activities related to homework/learning) (WHO 2017). However, recent evidence suggests that most children fail to meet these guidelines. For example, pooled data from the International Children's Accelerometry Database (ICAD), an international consortium of accelerometry data, combined results from Europe, Australia, North America, and South America. Longitudinal results from 1997 to 2009 found that only $9 \%$ of boys and $2 \%$ of girls aged 5 to 17 years achieved the recommended 60 minutes of MVPA every day (Cooper 2015). Likewise, the Active Healthy Kids Global Alliance (Aubert 2018), which involves children from 49 countries of varying human development index, found that only $20 \%$ of children aged 5 to 17 achieved the recommended amount of PA based on the WHO guidelines (Aubert 2018). This result is estimated to be lower for children from low socio-economic status (SES), with research demonstrating the lower the SES, the higher the levels of sedentary behaviour and lower levels of MVPA (Drenowatz 2010). To date, many children's PA interventions have been delivered in the school setting, with efforts aimed at improving the school curriculum to include PA as part of traditional learning, specific physical education lessons, and changes to the school environment or school policies, or both (Atkin 2011). However, delivery in the school setting has limitations. For example, it appears that behaviour changes achieved at school are not carried through to the afterschool period (Atkin 2011). In addition, overcrowding of the school curriculum has resulted in declining willingness for schools to take on PA interventions (Beets 2009). In contrast, the outsideschool hours period (before and after school) presents a relatively underexplored, and potentially more flexible, time period in which to intervene on children's PA.

The outside-school hours period refers to the hours of the day before and after the structured school day, typically between 7.00 a.m. and 8.30 a.m. in the morning and 3.00 p.m. and 6.00 p.m. in the afternoon, although this varies across countries. During these hours, children may be home or have the opportunity to participate in organised activities such as sports, scouts, dancing, art lessons, etc., or children may attend formalised childcare (e.g. outside school hours care (OSHC) in Australia or afterschool programmes (ASP) in the USA). There is a global shift in adults' work practices that has affected where and what school-aged children do during the outside-school hours period. Figures from the Organisation for Economic Co-operation and Development (OECD), which combined economic and social well-being data from 36 countries across 5 continents, showed that, on average, in 2016, across OECD countries $56 \%$ of children live in households where all adults are in work (combination of full time and part time); $34 \%$ have one adult in work; and just under $10 \%$ reside in a household where no adult works. This is in stark contrast to historic data from the OECD in 1999, in which only $30 \%$ of all adults worked. Hence, the increased need for formalised outside-school hours childcare has become a global phenomenon (OECD 2016). The OECD data from 2016 suggest that approximately $28 \%$ of all 6- to 11-year-old children attending school attend outside-school hours childcare. The rate is higher in Nordic countries such as Denmark and Sweden, with outside-school hours childcare use at $60 \%$, which coincides with both parents working in $70 \%$ of households. In contrast, the use of outside-school hours childcare is estimated to be as low as $6 \%$ to $8 \%$ in some Southern European countries (Italy and Spain) (OECD 2018). In the USA in 2014 it was estimated that 10.2 million, or $18 \%$, of school-aged children attended an afterschool childcare programme (Afterschool Alliance 2014). This has steadily risen from $11 \%$ in 2004 to $15 \%$ in 2009. Similar trends have been demonstrated in Australia, with approximately $10 \%$ of school-aged children between the ages of 5 and 12 years attending afterschool childcare services in 2017, compared to $8 \%$ in 2011 (ABS 2017). The increased use of such services suggests that outside-school hours childcare is an increasingly important setting in which to deliver PA programmes to address inactivity.

There have been no attempts to date to synthesise evidence regarding physical activity interventions delivered in the outside-school hours childcare setting. However, there have been two related systematic reviews both of which examined the efficacy of afterschool PA programmes. A 2011 review by Atkin and colleagues included nine studies, of which three positively impacted PA, and six found no change. Results suggested that programmes specifically target-

Interventions in outside-school hours childcare settings for promoting physical activity amongst schoolchildren aged 4 to 12 years 
ing PA (as opposed to targeting PA in concert with other health behaviours such as diet) may be more effective (Atkin 2011). Mears and Jago's 2016 review found 15 studies, of which six were included in a meta-analysis (Mears 2016). Again, the evidence for programme efficacy was mixed, with a small pooled intervention effect of just 5 minutes per day increased MVPA (Mears 2016). However, these reviews only incorporate data up to early 2015, and examined programmes delivered in the afterschool period generally, rather than in the afterschool childcare setting specifically. A contemporary and more focused review of the evidence is therefore warranted.

\section{Description of the intervention}

For the purpose of this review, the intervention of interest is any programme delivered in the outside-school hours childcare setting (i.e. consistent childcare programmes provided in the hours either before and/or after school and/or during the school holiday period) that aims to increase PA. We anticipate a variety of intervention approaches, including programmes focused solely on PA as well as those intervening on PA in conjunction with other behaviours (e.g. diet). In addition, programmes may include staff training in facilitation of active play, timetabling changes, provision of equipment for active play, provision of activities for active play, teaching physical literacy, or changes in policies at either a district, state, or national level.

\section{How the intervention might work}

The PA intervention may work through a variety of pathways, such as increasing opportunity for children to engage in PA (e.g. through timetabling changes with increased time allocated to outdoor play, or through provision of equipment that facilitates active play); increasing the intensity of activity during existing active playtime (e.g. by staff training that assists staff to run games that encourage children to be more vigorously active and/or improve children's physical literacy, i.e. the knowledge, motivation, and skills to be active); or by removing competing activities that discourage children from being active (e.g. removing or limiting access to recreational screen devices and other sedentary pursuits). Policies may be implemented, as in North America where the YMCA adopted the Healthy Eating and Physical Activity Standards (HEPA), which states that children must achieve at least 30 minutes of MVPA daily in the afterschool programme, as a way to help children achieve at least half of their recommended daily MVPA requirements (Beets 2018). This is similar in other countries where guidelines for physical activity in the afterschool childcare setting exist and are implemented as part of standard care (e.g. the Ontario Ministry for Education - Canada) (Ontario Ministry of Education 2017).

\section{Why it is important to do this review}

Given the significant health and economic impacts of physical inactivity globally, interventions are needed to address and improve this issue. More than 1 in 4 children internationally attend outside-school hours childcare programmes, and rates are rising, suggesting that this presents an increasingly important setting for children's PA interventions. There have been no systematic reviews on PA interventions in the outside-school hours childcare setting to date. Much of the existing evidence comes from short-term afterschool programmes (e.g. summer camps) set up by researchers to deliver targeted PA interventions for specific populations (e.g. African-American girls or overweight children) (Baranowski 2003; Barbeau 2007; Weintraub 2008). The findings of such studies may not be generalised to outside-school hours childcare settings, which service more diverse populations and operate on an ongoing basis in different contexts. However, there is increased policy and programme interest by governments and other agencies in this setting as an opportunity to promote child health. To guide such policy and practice decision, a systematic review synthesising the effects of interventions delivered in the school-aged childcare setting is required.

\section{O B J E C T I VES}

The primary objective is to assess the effects of physical activity interventions for increasing physical activity in children aged 4 to 12 years in outside-school hours childcare settings (i.e. the hours of the day when formal school lessons have either not begun or have finished and/or during school holiday periods). Our secondary objectives are to assess the effects of interventions on markers of children's cardiovascular health, quality of life, process evaluation and to identify any unintended adverse outcomes (e.g. injuries, distress).

\section{METHODS}

\section{Criteria for considering studies for this review}

\section{Types of studies}

Based on the criteria from the Cochrane Effective Practice and Organisation of Care Group (EPOC), we will include randomised controlled trials (RCTs) (including cluster-RCTs) in the review. We will exclude study designs using non-random assignment to groups. There will be no exclusions based on sample size, language, or country. 


\section{Types of participants}

Participants will be primary/elementary school-aged children who are attending outside-school hours childcare services, where the children are predominantly aged 4 to 12 years. In instances where the study includes a wider age range, these studies will still be included, however only the data for which the mean age of the participants is between 4 to 12 years will be included for analysis (authors will be contacted and raw data requested). For the purpose of this review, 'outside-school hours childcare services' are defined as formal, structured, group childcare services that operate prior to and/or following the usual school operational hours on days when children attend school. They may also occur during school holidays. They will include services that are provided either by a school or third-party provider such as community organisations or for-profit private providers. They will not include programmes provided by sporting clubs (e.g. soccer training during the week for weekend games) or other specific activity groups (e.g. gymnastics/ scouts/dance clubs) or temporary PA programmes offered outside of school terms (e.g. dance programmes set up for the school holiday period).

\section{Types of interventions}

Any intervention aimed at increasing PA levels in the outsideschool hours childcare setting will be eligible. Examples may include changes to activity schedules to increase opportunity for PA, environmental interventions (e.g. increasing the availability of activity play equipment, or access to areas conducive to PA); facilitated activities aimed at increasing PA (e.g. games or sports led by a facilitator); or regulatory interventions (e.g. implementation of a policy at either a district, state, or national level related to PA). We will include interventions that focus solely on increasing PA, and those that aim to increase PA in addition to affecting other health behaviours (e.g. improved diet) or health conditions (e.g. obesity).

To be eligible, the interventions must be delivered in the context of an existing outside-school hours childcare setting (i.e. childcare that is available consistently throughout the school week/ year). The childcare service may be operated by schools, community groups or non-government organisations (e.g. YMCA), or third-party providers (e.g. private childcare companies) in either a school, community setting, or childcare-specific facility. We will include interventions that involve strategies targeting physical activity in other contexts, such as the home or school, only if two review authors (RV and CM/LL/AP) judge the majority of the intervention to have occurred in the outside-school hours setting. Programmes based in a clinical setting (e.g. hospital-based exercise programme after school for weight loss) will be ineligible.

Comparisons will be usual care (i.e. outside-school hours childcare with no PA intervention); attention-matched control groups (i.e. where the control group receives a placebo technique to mimic time and attention given to intervention group participants); wait- list control groups (where the control group receives the intervention after the active intervention group); or alternative interventions (i.e. where a study compares two alternative forms of PA intervention).

\section{Types of outcome measures}

\section{Primary outcomes}

- Duration (minutes or \% time) MVPA as measured by direct observation (using a standardised, validated directobservation tool), accelerometry, self-report (i.e. from children or childcare staff or both, reported using a validated questionnaire), or heart rate monitor (where results have been extrapolated to minutes in MVPA). Data may be expressed in terms of baseline and postintervention mean values (and standard deviations) or change scores.

- Number of steps assessed by pedometry. Data may be expressed in terms of baseline and postintervention mean values (and standard deviations) or change scores.

- Proportion of care session spent in MVPA, assessed through either direct observation, accelerometry, or self-report . Data may be expressed in terms of baseline and postintervention percentages, or per cent change scores.

- Proportion of children categorised as being sufficiently active, assessed either through direct observation, accelerometry, or self-report. Data may be expressed in terms of baseline and postintervention proportions, or per cent change scores.

\section{Secondary outcomes}

- Cardiovascular health

- $\quad$ Cardiovascular fitness (e.g. 20-metre shuttle run test)

- Blood pressure

- Body mass index (BMI) (both self-report and objectively measured height and weight for BMI calculation)

- Quality of life measures (based on results from validated measurement tools, e.g. Pediatric Quality of Life Inventory (PedsQoL) and KINDL)

- Evaluation outcomes

- Process evaluation

- Cost evaluation

- Feasibility (which may be referred to as acceptability or feasibility)

- Adverse outcomes

- Any measures of adverse effects identified by studies including musculoskeletal injuries, or any psychological distress as a result of interventions

The outcomes (primary and secondary) will be prioritised into the following order from critical to important for use in the Summary of findings table: 
1. Intensity of Physical Activity (e.g. Sed, Light PA, MVPA) (Primary outcomes: number of steps, proportion of care session, categorization)

2. Overall Physical activity (Primary outcomes: duration of activity as minutes or $\%$ time)

3. Cardiovascular health (including secondary outcomes cardiovascular fitness, blood pressure, BMI)

4. Quality of Life (secondary outcome)

5. Adverse outcomes (secondary outcome)

\section{Search methods for identification of studies}

\section{Electronic searches}

We will search the following:

- the Cochrane Library (including the Cochrane Central

Register of Controlled Trials (CENTRAL));

- MEDLINE via Ovid platform;

- Embase via Ovid platform;

- ERIC (Education Resources Information Center);

- SportDISCUS.

We will use the search strategy for MEDLINE shown in Appendix 1 , which will be modified for other databases. There will be no language or date limits.

\section{Searching other resources}

We will search for grey literature in Trove and ProQuest Dissertations and Theses Global databases. We will conduct handsearches of the reference lists of included articles and citation tracking (forward citing) to identify relevant articles. We will handsearch all publications in the previous three years for two relevant journals. The key journals will be those in which included articles were most frequently published. Finally, we will contact first and senior authors on articles identified for inclusion for ongoing or unpublished research, using the contact details provided in the publication.

\section{Data collection and analysis}

\section{Selection of studies}

All references identified through the search strategy will be downloaded into reference management software Endnote Version X8 (EndNote 2018), which will remove duplicate references. These results will then be imported into Covidence for screening (Covidence 2018). Two review authors (RV and AP/LL/CM) will initially screen references for potential inclusion based on title and abstract. Review authors will not be blinded to study author or journal. A third review author will mediate any disagreements until consensus is reached. We will obtain the full texts of studies deemed potentially eligible for inclusion, and two review authors (RV and CM) will assess the full texts for eligibility against the inclusion criteria. Those studies deemed as ineligible will be recorded with reasons for ineligibility in the 'Characteristics of excluded studies' table. Any disagreements will be resolved by consulting a third review author (LL) as necessary. Regarding any papers for which detail to determine the eligibility is lacking, we will contact the study author directly. We will create a flow chart following PRISMA protocol to illustrate this process.

\section{Data extraction and management}

Two review authors (RV and KB) will extract data onto piloted pro forma developed for this review (see Appendix 2). In case of disagreement, a third review author (CM) will mediate to reach consensus. We will attempt to contact authors directly, recording date and method of contact if the information required for data extraction is not available from the published report or is unclear. We will extract the following data.

- Background and general information

- Date of extraction, review author ID, title, published or unpublished, authors, years of publication, country, contact address, study country, language of publication, source of study, funding, conflicts of interest

- Basic study details

- Sample size, participant characteristics, attrition rates

- Population and setting

- Description of population and setting, characteristics of participants (age, gender, location, SES, etc.), recruitment strategy

- Methods

- Aim of intervention, allocation method, number of study arms, description of study arms, sample size per study arm, start date, end date, duration of participation

- Participants

- Total number randomised; presence or not of baseline imbalances; if there is an imbalance, description of such; number of withdrawals/exclusions; sex of children; mean age; race/ ethnicity; SES

- Intervention group

- Number randomised, number measured at baseline, number measured at follow-up, intervention details, duration of intervention, delivery/providers of intervention

- Comparison group

- Number randomised, number measured at baseline, number measured at follow-up, comparison/usual care details, delivery/providers of comparison

\section{- Outcomes}

- For each outcome: measurement tool, psychometric properties of tool, assessor (i.e. person who performed 
assessments), whether missing data were imputed, units

- Other relevant information

○ Cost of intervention (if available), reported

limitations, process evaluation, other as appropriate

\section{Assessment of risk of bias in included studies}

Risk of bias for each review outcome will be assessed by two independent reviewers (RV and AP/CM/LL) using the Cochrane'Risk of bias' tool as described in the Cochrane Handbook for Systematic Reviews of Interventions (Higgins 2011). For each outcome within an included study we will assess the following risk of bias domains: selection bias (random sequence generation and allocation concealment), performance bias (blinding of participants and personnel), detection bias (blinding of outcome assessment), attrition bias (incomplete outcome data), reporting bias (selective reporting) and other bias (other sources of bias), providing a rating of low, high, or unclear risk of bias. For cluster-RCTs, we will also assess the following domains as per the Cochrane Handbook for Systematic Reviews of Interventions: recruitment bias, baseline imbalances, loss of clusters, incorrect analysis (Higgins 2011).

We will designate an overall risk of bias for an outcome within a study (across domains). It is unlikely that blinding of participants and personnel will be possible for the interventions examined. Therefore we will assign a high risk of bias to a study when the trial is judged to be at high risk of bias for an outcome on more than one of the following key criteria: sequence generation (selection bias), allocation sequence concealment (selection bias), incomplete outcome data (attrition bias), and in instances where self-report measures of outcome are employed blinding of outcome assessment.

We will assign a low risk of bias to a study when the trial is judged to be at low risk of bias for an outcome on all key criteria. Risk of bias will also be summarised for an outcome across studies. We will judge an outcomes as i) low risk if most information for the outcome is generated from studies at low risk of bias ii) unclear risk of bias if most information is from studies at low or unclear risk of bias; or iii) high risk of bias if the proportion of information from studies at high risk of bias is sufficient to affect the interpretation of results. Such judgements will be made independently by two reviewer authors and any discrepancies resolved via consensus.

\section{Measures of treatment effect}

We will use Review Manager 5 to manage data and complete the analysis (RevMan 2014). We will report effect sizes alongside $95 \%$ confidence intervals (CIs). We expect that the primary outcomes will be assessed using several different measures (i.e. time in MVPA, \% session in MVPA), and we will combine and pool measures where possible.

\section{Dichotomous data}

For dichotomous measures of PA (primary outcomes), we will calculate the risk ratio (RR) using the proportion of events in the intervention group as the numerator and the proportion of events in the control group as the denominator.

\section{Continuous data}

For continuous measures of PA (primary outcome), cardiovascular health (secondary outcome), and quality of life (secondary outcome), we will report the mean difference (MD) for the results per outcome, that is the difference in the mean change between the intervention and control group. We expect that the data for a given outcome may be reported using different tools/measures, in which case we will use the standardised mean difference (SMD).

\section{Categorical data}

Where possible, we will collapse categorical data into a small number of categories and analyse as dichotomous data.

We will synthesise a narrative summary for evaluation outcomes and adverse events (secondary outcomes).

\section{Unit of analysis issues}

\section{Cluster-RCTs}

We will examine all cluster-RCTs for unit of analysis errors and document any that are identified in the 'Risk of bias' tables. We will follow the recommendations in the Cochrane Handbook for Systematic Reviews of Interventions for cluster-RCTs (Higgins 2011), that is where the study authors provide a direct estimate of the required effect (e.g. an odds ratio and confidence interval) from an analysis that properly accounts for the cluster design, it will be included in the meta-analysis. Where cluster-RCTs do not account for clustering, we will contact the study authors for intraclass correlation coefficients (ICCs) in order to calculate design effects and effective sample sizes to enable individual-level pooling. Where ICCs are not available, we will estimate a mean ICC from reported ICCs of included studies, and use it to calculate effective sample sizes.

\section{Repeated measures}

In the event that repeated measures are taken, that is long-term follow-up, we will select the longest follow-up for analysis as per the Cochrane Handbook for Systematic Reviews of Interventions (Higgins 2011). 


\section{Multiple intervention groups}

For studies with multiple intervention groups, we will combine groups, that is intervention groups versus control groups, to create a single pairwise comparison, using the same methods of analysis as described for dichotomous or continuous data as appropriate.

\section{Dealing with missing data}

In the case of missing data, we will first contact the original investigator for the missing data where possible (e.g. when a study is identified as abstract only), as recommended in the Cochrane Handbook for Systematic Reviews of Interventions (Higgins 2011). Alternatively, we will calculate missing standard deviations from other statistics such as standard errors, CIs, or P values, according to the methods outlined in the Cochrane Handbook for Systematic Reviews of Interventions (Higgins 2011). We will not impute any other missing values. We would address the likely impact of the missing data and the replacement values on the results of the metaanalysis as part of the Discussion section of the review.

\section{Assessment of heterogeneity}

We will assess heterogeneity via visual inspection of forest plots, $\mathrm{Chi}^{2}$ test, and the $\mathrm{I}^{2}$ statistic, as per the Cochrane Handbook for Systematic Reviews of Interventions (Higgins 2011). We will use the $\mathrm{I}^{2}$ statistic to quantify heterogeneity among the trials in each analysis. If we find considerable heterogeneity, we will report it and explore possible causes by prespecified subgroup analysis.

We will follow the threshold recommendations outlined in Section 9.5.2 of the Cochrane Handbook for Systematic Reviews of Interventions (Higgins 2011):

- $0 \%$ to $40 \%$ : might not be important;

- $30 \%$ to $60 \%$ : moderate heterogeneity;

- $50 \%$ to $90 \%$ : substantial heterogeneity;

- $75 \%$ to $100 \%$ : considerable heterogeneity.

We will investigate heterogeneity through subgroup analyses, and we will use this information to make decisions as to whether to conduct meta-analysis (Higgins 2011).

\section{Assessment of reporting biases}

We will use funnel plots to provide visual representation of the intervention effect estimates. If the plot is asymmetrical, this may identify publication bias, poor study design, or the effect of study size. Additionally, we will compare registers/protocols of included studies with final reports to further identify reporting biases.

\section{Data synthesis}

We anticipate that the outcomes of interest will be measured using a variety of measures and reported using various methods, for example percentage of the session spent in MVPA or percentage of children categorised as sufficiently active or inactive. We will attempt to conduct meta-analysis of measures of:

- intensity of activity (MVPA) (time (minutes) in MVPA, \% time in MVPA);

- overall activity (steps, total counts, etc.);

- fitness;

- blood pressure;

- weight status/BMI.

We will conduct the analyses using Review Manager 5 software (RevMan 2014), employing inverse-variance and random-effects models. Random-effects models are based on the assumption that the true effect might vary from study to study. The CIs for the average intervention effect results from a random-effects model will be wider than those obtained using a fixed-effect approach, leading to a more conservative interpretation.

We will use estimates of intervention effect and standard error to calculate the effect size. Where possible, that is the data collected are in the same format, we will conduct a meta-analysis. Where the data are heterogenous, we will perform a narrative synthesis accompanied by a summary table including such details as the number of studies, the interventions, and the different outcome measures used.

We will synthesise data regarding evaluation outcomes (such as process evaluation, intervention cost and feasibility) and adverse events narratively.

We will consider the clinical significance of results for the primary outcome (PA) in the context of whole-day PA, that is whether the effect of any increase in MVPA is of sufficient magnitude to substantially contribute to achievement of WHO-recommended daily MVPA of 60 minutes for children aged 5 to 17 years.

\section{Subgroup analysis and investigation of heterogeneity}

Where data allow, we will conduct subgroup analyses to explore heterogeneity across studies for the primary outcome of MVPA based upon:

- intervention (short term $<3$ months versus long term $>12$ months);

- population (e.g. schools for children with disability versus mainstream);

- age (younger children age 4 to 7 years versus older children 8 to 12 years);

- outcome (e.g. BMI).

\section{Sensitivity analysis}

Where appropriate, we will perform sensitivity analyses and report the results in a summary table. This will likely include:

- influence of clustering: removing cluster-RCTs that did not account for clustering;

- influence of risk of methodological bias: removing those studies assessed as at high risk of bias. 
We will not perform sensitivity analysis on source of funding; this will be addressed during the 'Risk of bias' assessment.

\section{'Summary of findings' table}

Two review authors (RV and CM/LL/AP) will use the GRADE approach to assess the quality of evidence for the outcomes that are addressed by the included studies (Guyatt 2013). The GRADE approach uses select criteria to determine the quality of the evidence by assessing the methodological quality at an outcome level, heterogeneity, directness of evidence, precision of evidence, and risk of publication bias (Guyatt 2013). The outcomes are graded as follows.

- High quality: further research is very unlikely to change our confidence in the effect estimate.

- Moderate quality: further research is likely to have an important impact on our confidence in the effect estimate and may change the estimate.

- Low quality: further research is very likely to have an important impact on our confidence in the effect estimate and may change the estimate.

- Very low quality: we are very uncertain about the effect estimate.

We will export data from Review Manager 5, RevMan 2014, into GRADEpro GDT software, GRADEpro GDT 2015, to produce a 'Summary of findings' table which can then be directly imported into Review Manager 5. These tables allow for details for each outcome assessed to be collated, such as the assessment tools used, follow-up range, timing of follow-up, study design, number of studies, total sample sizes, effect estimates, and quality of the evidence. Any disagreements with be resolved through discussion with a third review author (CM/LL/AP). The following outcomes (primary and secondary) will be prioritised into the following order from critical to important for use in the Summary of findings table:

1. Intensity of Physical Activity (e.g. Sed, Light PA, MVPA) (Primary outcomes: number of steps, proportion of care session, categorization)

2. Overall Physical activity (Primary outcomes: duration of activity as minutes or $\%$ time)

3. Cardiovascular health (including secondary outcomes cardiovascular fitness, blood pressure, BMI)

4. Quality of Life (secondary outcome)

5. Adverse outcomes (secondary outcome)

For ease of interpretation of the standardised effect sizes, we will apply the following rules in accordance with the Cochrane Handbook for Systematic Reviews of Interventions (Higgins 2011), where a standard deviation of 0.2 represents a small difference between groups; 0.5 represents a moderate difference; and 0.8 represents a large difference.

\section{ACKNOWLEDGEMENTS}

We acknowledge the help and support of Jodie Doyle (Managing Editor) for Cochrane Public Health group. We acknowledge the help of Carole Gibbs and Sarah McQuillen, academic librarians from the University of South Australia, for their assistance with the search strategy.

\section{R E F E R E N C E S}

\section{Additional references}

ABS 2017

Australian Bureau of Statistics. Childhood education and care Australia. Canberra (ACT): Australian Bureau of Statistics; 2017 June. Report No.:44020DO001_201706.

Afterschool Alliance 2014

Afterschool Alliance. America after 3pm: afterschool programs in demand. Washington (DC): Afterschool Alliance; 2014.

\section{Atkin 2011}

Atkin AJ, Gorely T, Biddle SJ, Cavill N, Foster C. Interventions to promote physical activity in young people conducted in the hours immediately after school: a systematic review. International Journal of Behavioural Medicine 2011;18(3):176-87.

\section{Aubert 2018}

Aubert S, Barnes JD, Abdeta C, Abi Nader P, Adeniyi AF, Aguilar-Farias N, et al. Global matrix 3.0 physical activity report card grades for children and youth: results and analysis from 49 countries. Journal of Physical Activity and Health 2018;15 Suppl 2:251-73.

Baranowski 2003

Baranowski T, Baranowski JC, Cullen KW, Thompson DI, Nicklas T, Zakeri IF, et al. The fun, food, and fitness project (FFFP): the Baylor GEMS pilot study. Ethnicity and Disease 2003;13 Suppl 1:1-30.

\section{Barbeau 2007}

Barbeau P, Johnson MH, Howe CA, Allison J, Davis CL, Gutin B, et al. Ten months of exercise improved general and visceral adiposity, bone and fitness in black girls. Obesity 2007;15(8):2077-85.

Bassett 2015

Bassett DR, John D, Conger SA, Fitzhugh EC, Coe DP. Trends in physical activity and sedentary behaviours of United States youth. Journal of Physical Activity and Health 2015;12(8):1102-11. DOI: 10.1123/jpah.2014-0050 
Beets 2009

Beets MW, Beighle A, Erwin HE, Huberty JL. After-school program impact on physical activity and fitness: a metaanalysis. American Journal of Preventive Medicine 2009;36 (6):527-37.

Beets 2018

Beets MW, Weaver GR, Brazendale K, Turner-McGrievy G, Saunders RP, Moore JB, et al. Statewide dissemination and implementation of physical activity standards in afterschool programs: two-year results. BMC Public Health 2018;18 (1):819-33.

Booth 2015

Booth VM, Rowlands AV, Dollman J. Physical activity temporal trends among children and adolescents. Journal of Science and Medicine in Sport 2015;18(4):418-25.

\section{Cooper 2015}

Cooper AR, Goodman A, Page AS, Sherar LB, Esliger DW, van Sluijs EM, et al. Objectively measured physical activity and sedentary time in youth: the International children's accelerometry database (ICAD). International Journal of Behavioural Nutrition and Physical Activity 2015;12(1):113.

Covidence 2018 [Computer program]

Veritas Health Innovation. Covidence. Version accessed January 2018. Melbourne, Australia: Veritas Health Innovation, 2018.

\section{Dalene 2018}

Dalene KE, Anderssen SA, Andersen LB, SteeneJohannessen J, Ekelund U, Hansen BH, et al. Secular and longitudinal physical activity changes in population-based samples of children and adolescents. Scandinavian Journal of Medicine and Science in Sports 2018;28(1):161-71.

Ding 2016

Ding D, Lawson KD, Kolbe-Akexader TL, Finkelstein EA, Katzmarzyk PT, van Mechelen W, et al. The economic burden of physical inactivity: a global analysis of major non-communicable diseases. Lancet 2016;388(10051): $1311-24$.

Dollman 2005

Dollman J, Norton K, Norton L. Evidence for secular trends in children's physical activity behaviour. British Journal of Sports Medicine 2005;39(12):892-7. DOI: 10.1136/ bjsm.2004.016675

Drenowatz 2010

Drenowatz C, Eisenmann JC, Pfeiffer KA, Welk G, Heelan $\mathrm{K}$, Gentile $\mathrm{D}$, et al. Influence of socio-economic status on habitual physical activity and sedentary behaviour in 8 to 11 year old children. BMC Public Health 2010;10(1):214. DOI: $10.1186 / 1471-2458-10-214$

EndNote 2018 [Computer program]

Clarivate Analytics. EndNote X9. Boston (MA): Clarivate Analytics, 2018.

GRADEpro GDT 2015 [Computer program] McMaster University (developed by Evidence Prime). GRADEpro GDT. Version accessed January 2019.
Hamilton (ON): McMaster University (developed by Evidence Prime), 2015.

\section{Guyatt 2013}

Guyatt G, Oxman AD, Sultan S, Brozek J, Glasziou P, Alonso-Coello P, et al. GRADE guidelines: 11: Making an overall rating of confidence in effect estimates for a single outcome and for all outcomes. Journal of Clinical Epidemiology 2013;66(2):151-7.

\section{Higgins 2011}

Higgins JP, Green S, editor(s). Cochrane Handbook for Systematic Reviews of Interventions Version 5.1.0 (updated March 2011). The Cochrane Collaboration, 2011. Available from handbook.cochrane.org.

\section{Jones 2013}

Jones RA, Hinkley T, Okely AD, Salmon J. Tracking physical activity and sedentary behavior in childhood: a systematic review. American Journal of Preventive Medicine 2013;44(6):651-8.

\section{Mears 2016}

Mears R, Jago R. Effectiveness of after-school interventions at increasing moderate-to-vigorous physical activity levels in 5- to 18-year olds: a systematic review and meta-analysis. British Journal of Sports Medicine 2016;50(21):1315-24.

\section{OECD 2016}

Organisation for Economic Co-operation and Development - Social policy division. LMF 2.2: Patterns of employment and the distribution of working hours for couples with children. Paris (IDF): Organisation for Economic Cooperation and Development (OECD); OECD Database; 2016. www.oecd.org/els/family/LMF-2-2-Distributionworking-hours-couple-households.pdf (accessed 10 February 2019).

\section{OECD 2018}

Organisation for Economic Co-operation and Development - Social policy division. PF4.3: Out-of-school-hours care services. Paris (IDF): Organisation for Economic Cooperation and Development (OECD); OECD Database; 2018. www.oecd.org/els/family/PF4-3-Out-of-schoolhours-care.pdf (accessed 10 February 2019).

Ontario Ministry of Education 2017

Ontario Ministry of Education. Before-and-after school programs (kindergarten to grade 6) policies and guidelines for school boards. Toronto (ON): Ontario Ministry of Education; 2017 January.

Reiner 2013

Reiner M, Niermann C, Jekauc D, Woll A. Long-term health benefits of physical activity - a systematic review of longitudinal studies. BMC Public Health 2013;13(1):813

\section{RevMan 2014 [Computer program]}

Nordic Cochrane Centre, The Cochrane Collaboration. Review Manager 5. Version 5.3. Copenhagen: Nordic Cochrane Centre, The Cochrane Collaboration, 2014. 


\section{Sterdt 2014}

Sterdt E, Liersch S, Walter U. Correlates of physical activity of children and adolescents: a systematic review of reviews. Health Education Journal 2014;73(1):72-89.

\section{Tammelin 2014}

Tammelin R, Yang X, Leskinen E, Kankaanpaa A, Hirvensalo M, Tammeline T, et al. Tracking of physical activity from early childhood through youth into adulthood. Medicine and Science in Sports and Exercise 2014;46:955-62.

\section{Weintraub 2008}

Weintraub DL, Tirumalai EC, Haydel KF, Fujimoto M, Fulton JE, Robinson TN. Team sports for overweight children: the Stanford sports to prevent obesity randomized trial (SPORT). Archives of Pediatric and Adolescent Medicine 2008;162(3):232-7.

\section{WHO 2017}

World Health Organization. Physical Activity and Young People: Recommended levels of physical activity for children aged 5-17 years; 2017. www.who.int/ncds/ prevention/physical-activity/factsheet_young-people/en/ 2017.

WHO 2018a

World Health Organization. Noncommunicable diseases and their risk factors; 2018. www.who.int/ncds/en/ 2018.

\section{WHO 2018b}

World Health Organization. Non communicable diseases and their risk factors - WHO launches ACTIVE: A toolkit for countries to increase physical activity and reduce non communicable disease; 2018 . www.who.int/ncds/ prevention/physical-activity/active-toolkit/en/ 2018

* Indicates the major publication for the study

\section{A P P E N D I C E S}

\section{Appendix I. Ovid MEDLINE search strategy}

1. exp Child/

2. school teacher/

3. (child ${ }^{*}$ or schoolchild ${ }^{*}$ or preschool* ${ }^{*}$ or school age* or schoolage* or schoolboy* ${ }^{*}$ or schoolgirl ${ }^{*}$ or boy? or girl? or teacher? or facilitator? or educator? or instructor?).mp.

4. 1 or 2 or 3

5. exp exercise/

6. motor activity/

7. exp physical fitness/

8. "Physical Education and Training"/

9. Walking/

10. Stair climbing/

11. exp Sports/

12. dancing/

13. exp Exercise Therapy/

14. (exercis* or physical* activ* or physical education or physical training or motor activity or fitness or aerobic? or walk* or stair climb* or climb* stair? or sport* or athletics or athletic performance or physical endurance or baseball or basketball or bicycling or cycling or boxing or football or golf or gymnastics or hockey or martial arts or tai ji or tai chi or mountaineering or tennis or badminton or run or running or ran or "track and field" or jog or jogging or jogged or cross country or orienteering or skating or skiing or volleyball or swim or swimming or swam or weight lifting or wrestling or dance or dancing or danced or yoga or active lifestyle).mp.

15. Or/5-14

16. (after school* or afterschool* or before school* or out of school* or vacation or holiday or OSHC or ASP).mp.

17. 4 and 15 and 16

18. randomized controlled trial.pt.

19. controlled clinical trial.pt.

20. randomized.ab.

21. placebo.ab.

22. clinical trials as topic.sh.

23.randomly.ab.

Interventions in outside-school hours childcare settings for promoting physical activity amongst schoolchildren aged 4 to $\mathbf{2}$ years 
24. trial.ti.

25.18 or 19 or 20 or 21 or 22 or 23 or 24

26. exp animals/ not humans.sh.

27. 25 not 26

\section{Appendix 2. Data extraction form}

Data extraction form - Cochrane Review

Form Version/Date: Version 1.0; 19 February 2019

Review Title: Interventions in outside-school hours settings for promoting physical activity amongst schoolchildren aged 4 to 12 years

Study: First author and year of publication:

Name of review author completing this form:

Date form completed:

Notes: Unpublished - for own use e.g. references to be followed up, source of information (especially if multiple reports of the same trial, or unpublished data/personal communication included)

Methods:

Notes/comments Paper/ page

Details of Study

Aim of intervention (As stated in the trial report/s. What was the problem that this intervention was designed to address?)

Aim of study (As stated in the trial report/s.

What was the trial designed to assess?)

Study design / i.e. CRCT or RCT; number of experimental conditions

Unit of randomisation (i.e. individual or group for cluster trials)

Method of randomisation 
(Continued)

Methods of recruitment of participants

(How were potential participants approached and invited to participate? ADDED: Brief description only here: e.g. Convenience. No need to contact authors if missing).

Inclusion/exclusion criteria for participation in study (ADDED: Include explicit criteria only, not only post-hoc exclusions).

Informed consent obtained? (Yes/No/Unclear)

Ethical approval (Yes/No/Unclear -no details required)

Funding (including source, amount, ifstated)

Study Date/Recruitment date

Consumer involvement (e.g. In design of study andlor intervention; in delivery of intervention; in evaluation of intervention; in interpretation of study findings)

Participants:

\section{Notes/comments Paper/ page}

Description (e.g. children; carers; parents of children; educators; policy makers) ADDED:

Choose from these categories only

Age: range, mean (SD)

Gender: \% Male, \% Female

SES: (disadvantaged population (yes/no/ unclear) e.g. mean income, maternal education

Geographic location (e.g. City/State/ Country) 
Setting (e.g. school based outside school hours care, community based outside school hours care

Presence of baseline imbalance

Intervention and Comparison:

Intervention A Control Paper/ page

Overview / content:

\section{Number:}

Assessed for eligibility:

Eligible:

Excluded:

Refused to take part:

Randomised:

Allocated:

Received allocated interven-

tion/control:

Did not receive allocated inter-

vention / control:

Lost to follow up:

Withdrawn/discontinued intv/

control:

Analysed:

Excluded from analysis:

\section{Theoretical basis}

(e.g. sociocognitive theory)

Delivery format / modality

\section{Setting}

(e.g. School based OSHC, community based, church/ private third party/government run)

\section{Delivery information:}

\section{Number of sessions:}

\section{Duration of intervention}

\section{Intervention period}

Interventions in outside-school hours childcare settings for promoting physical activity amongst schoolchildren aged 4 to 12 years

(Protocol)

Copyright $\Subset 2019$ The Cochrane Collaboration. Published by John Wiley \& Sons, Ltd. 
(Continued)

Details of providers:

Who delivers the intervention

Number of providers

Training received in intervention delivery

Qualifications of intervention providers

Process measures:

Was the intervention delivered as intended (Record any assessment of this).

Details of co-interventions

Outcomes:

Notes/comments Paper/page

Principal and secondary outcome measures (as identified by the study authors).

Only include if relevant to the review. Note if NOT primary outcome).

Methods of assessing outcome measures (e. g. accelerometry, pedometers, self-report questionnaire)

Validity and reliability of outcome measures reported? If so, paste quote from paper in here

Methods of follow-up for non-respondents

Timing of outcome assessment (including frequency, length of follow up (for each outcome))

Adverse events (e.g. complaints, levels of dissatisfaction, adverse incidents, side effects)) if presence or absence of events not clearly stated = 'unclear'

Interventions in outside-school hours childcare settings for promoting physical activity amongst schoolchildren aged 4 to 12 years

(Protocol)

Copyright (๑) 2019 The Cochrane Collaboration. Published by John Wiley \& Sons, Ltd. 
(Continued)

Statistical analysis (include method and any assumptions made for intention to treat analyses and for CRCT any accounting for clustering effects

Unit of analysis

\section{Results}

These data will be used in the 'Comparisons and data' section in Review Manager 5 (not the 'Characteristics of included studies' table) and as the basis for the Results section of your review text.

All data are numbers (of participants), not percentages.

Dichotomous outcomes

\begin{tabular}{l|l|l|l|l}
\hline \multirow{2}{*}{$\begin{array}{l}\text { Outcome } \\
\text { come assessment } \\
\text { (days/months) }\end{array}$} & Observed (n) & Total (N) & Observed (n) & Total (N) \\
\cline { 2 - 5 } & & & Control group & \\
\hline & & & \\
\hline
\end{tabular}

${ }^{*}$ Note: add additional columns if there is more than one intervention group, e.g. Intervention Group A, Intervention Group B...

Any other reported results (i.e. effect estimates, $\mathrm{P}$ values)

\section{Continuous outcomes}




\begin{tabular}{|c|c|c|c|c|c|c|c|c|}
\hline \multirow[t]{2}{*}{ Outcome } & \multirow{2}{*}{$\begin{array}{l}\text { Tim- } \\
\text { ing of out- } \\
\text { come assess- } \\
\text { ment (days/ } \\
\text { months) }\end{array}$} & \multicolumn{3}{|c|}{ Intervention group } & \multicolumn{3}{|l|}{ Control group } & \multirow[t]{2}{*}{ Notes } \\
\hline & & $\begin{array}{l}{ }^{*} \text { Mean (pre- } \\
\text { post) / Mean } \\
\text { change }\end{array}$ & $\begin{array}{l}\text { Standard de- } \\
\text { viation }\end{array}$ & $\mathrm{N}$ & $\begin{array}{l}{ }^{*} \text { Mean (pre- } \\
\text { post) / Mean } \\
\text { change }\end{array}$ & $\begin{array}{l}\text { Standard devia- } \\
\text { tion }\end{array}$ & $\mathrm{N}$ & \\
\hline & & & & & & & & \\
\hline & & & & & & & & \\
\hline & & & & & & & & \\
\hline
\end{tabular}

Any other reported results (i.e. effect estimates, P values)

*Delete as appropriate

\section{CONTRIBUTIONS OFAUTHORS}

$\mathrm{RV}, \mathrm{CM}, \mathrm{LL}, \mathrm{AP}$ and MR conceptualised the review question.

All authors contributed to the writing of the protocol. RV and CM focused on the Background section, while RV and LW focused on the Methods section.

RV developed the search strategy with input from CM, LW, AP, and LL and academic librarians from the University of South Australia.

RV, CM, LL, AP, and KB will be responsible for searching for studies and data extraction and analysis. All authors will contribute to analysing the Results and Discussion sections.

RV and CM have overall responsibility for the review.

\section{DECLARATIONSOF INTEREST}

Luke Wolfenden is a Co-ordinating Editor for Cochrane Public Health Group.

Mandy Richardson is an Out of School Hours Care Director for St Pius X School, Windsor Gardens SA, Australia. In addition, she is the South Australian Chairperson for the Out of School Hours Care Assocaition and the South Australian Representative for the National Out of School Hours Care Services Alliance and a member of the Education Standards Board of South Australia.

Rosa Virgara, Carol Maher, Lucy Lewis, Anna Phillips, Anthony Okely, Michael Beets and Katherine Baldock have no known declarations of interest. 


\section{SOURCES OF SUPPORT}

\section{Internal sources}

- University of South Australia, Other.

Associate Professor Carol Maher, Dr Anna Phillips, Dr Katherine Baldock, and Ms Carole Gibbs are supported by the University of South Australia employment

- Flinders University, Other.

Dr Lucy Lewis is supported through Flinders University through employment.

- University of Wollongong, Other.

Professor Anthony Okely is supported through employment at the University of Wollongong

- University of South Carolina, Other.

Professor Michael Beets is supported through employment at University of South Carolina

- St Pius X School, Other.

Ms Mandy Richardson is supported by employment at St Pius X School.

- Hunter New England Health and University of Newcastle, Other.

Associate Professor Luke Wolfenden is supported through employment at Hunter New England Health and University of Newcastle

\section{External sources}

- NHMRC, Australia.

Associate Professor Carol Maher is supported by an NHMRC Career Development Position (2017-2020)

- University of South Australia - Research Training Program domestic (RTPd) Stipend. Health Development Adelaide, University of Adelaide - PhD Top Up Scholarship, Australia.

Ms Rosa Virgara is a PhD Candidate with the University of South Australia and is supported by a RTPd Stipend. In addition she is supported by a Top Up Scholarship from Health Development Adelaide, University of Adelaide

- NHMRC, Australia.

Associate Professor Luke Wolfenden is an NHMRC Career Development Fellow (2013 to present) CDF II and Brawn Career Development Fellow at School of Medicine and Public Health, University of Newcastle. He is also Hunter New England Clinical Research Fellow for Hunter New England Population Health. 